\title{
Strangers and Friends: Collaborative Play in World of Warcraft
}

\author{
Bonnie Nardi and Justin Harris \\ Department of Informatics \\ Donald Bren School of Information and Computer Sciences \\ University of California, Irvine \\ 1 (949) 824-6534 \\ \{nardi, jharris2\}@ics.uci.edu
}

\begin{abstract}
We analyze collaborative play in an online video game, World of Warcraft, the most popular personal computer game in the United States, with significant markets in Asia and Europe. Based on an immersive ethnographic study, we describe how the social organization of the game and player culture affect players' enjoyment and learning of the game. We discovered that play is characterized by a multiplicity of collaborations from brief informal encounters to highly organized play in structured groups. The variety of collaborations makes the game more fun and provides rich learning opportunities. We contrast these varied collaborations, including those with strangers, to the "gold standard" of Gemeinschaft-like communities of close relations in tightknit groups. We suggest populations for whom similar games could be designed.
\end{abstract}

\section{Categories and Subject Descriptors}

H.5.3 [Group and Organization Interfaces]: Synchronous Interaction

\section{General Terms}

Design, Human Factors

\section{Keywords}

Multiplayer games, MMOGs, play, social ties, community, Gemeinschaft, social isolation, learning

\section{INTRODUCTION}

Understanding changing social relations as they are developing within the context of the Internet is an important task for CSCW research [3]. A new and rapidly growing site of online collaboration is massively multiplayer online games (MMOGs). MMOGs are part of a larger group of video games that has surpassed film in box office revenue, with MMOGs accounting for about half total game revenue [16]. Blizzard

Permission to make digital or hard copies of all or part of this work for personal or classroom use is granted without fee provided that copies are not made or distributed for profit or commercial advantage and that copies bear this notice and the full citation on the first page. To copy otherwise, or republish, to post on servers or to redistribute to lists, requires prior specific permission and/or a fee.

CSCW'06, November 4ñ8, 2006, Banff, Alberta, Canada.

Copyright 2006 ACM 1-59593-249-6/06/0011 ...\$5.00
Entertainment's World of Warcraft is the best-selling PC title of any genre in the U.S., with 6.5 million active subscribers, including one million in Europe and 1.5 million in China [2]. Released in November 2004, World of Warcraft's rapid growth surprised everyone including Blizzard [25].

MMOGs connect hundreds or thousands of players through the Internet in persistent game worlds. Brown and Bell [3] observed that multiplayer games are one of the most collaborative and popular computer-based applications, with appreciative audiences all over the world. In this paper we describe diverse types of collaborative play in World of Warcraft, ranging from lightweight encounters with strangers to highly organized groupings with well-known friends. We argue that together these collaborations constitute a distinctive space of play made possible by the capabilities of the Internet, the design of World of Warcraft, and the culture created by players. Many collaborations spontaneously take place with strangers - a striking phenomenon that seems unusually prevalent in multiplayer games and suggests the emergence of new kinds of social relations developing within contexts provided by the Internet [3]. At the same time, many play WoW (as it is known) with offline friends and family, so the game also appears to reinforce existing social ties for these players.

Now that games are an important part of Internet use, we have an opportunity to recognize the collaborative aspects of online fun, to expand "CSCW" to include activities that provide entertainment $[3,6,8,9,12,19,30,35]$. Though the outcome of such activities is amusement and not a work product, there is a collective object-oriented activity [18], i.e., having fun. Even when players are competing, the object of having fun by playing the game is a form of collaboration; players could not compete if there were no one to play with. As in any game or sport, the larger field of collaboration is constituted by engaging in the game.

\section{WORLD OF WARCRAFT}

Based on a Tolkienesque high fantasy motif, World of Warcraft is a MMOG in which players create characters with distinctive looks and qualities such as intellect, strength, stamina, and agility. Characters advance through 60 levels of play. They acquire equipment such as swords, armor, and jewelry; develop skills such as attacking and healing; and slay an array of imaginative monsters dwelling in caves, dungeons, and encampments in varied landscapes in the land of Azeroth. The game can be likened to an animated cartoon in which the 
player controls the character with mouse and keyboard. Characters travel on foot, by boat or air, through fields, farms, deserts, mountains, seas, and other distinctive scenery for which WoW is known [34]. Lyrical or haunting music often plays as the character adventures.

Players are divided into "realms" which are servers of about 20,000 players [33]. The first key decision a player makes is which race and class to play. The "races" in WoW are night elf, dwarf, gnome, human, troll, orc, tauren, and undead. Classes are druid, priest, warrior, rogue, mage, hunter, paladin, shaman, and warlock (see Figure One). Each class entails a different style of play - for example, the priest is physically weak but able to cast deadly spells or heal fellow players while the warrior can take a beating from powerful monsters, keeping them at bay as other players struggle to defeat them. The classes' asymmetrical strengths and weaknesses encourage collaboration during battle as well as in brief encounters, such as asking a rogue to use his lock picking ability to open a chest buried at sea, or requesting conjured water from a mage.

World of Warcraft is a game comprised of killing monsters, exploration, quests, and battlegrounds in addition to more restful pursuits such as crafting, chatting with friends, and spending time in cities for banking, training, and trading. In a quest, the key form of play, a computer character gives the player a goal, for example, slaying the traitorous Edwin VanCleef. The player must travel to a certain area, kill a series of monsters, and finally battle VanCleef. Once the quest is completed, the player receives "experience points" which count toward reaching the next level of play, and possibly a reward such as equipment, in-game money, or fanciful items like a bouquet of flowers or a flagon of beer.

Game activities include practicing professions such as herbalism or blacksmithing, buying and selling items at the Auction House or through a trade chat channel, and socializing in many venues. Once level 60 is reached, the game goes on in dungeons with lengthy quests that offer the chance to acquire "epic" and "legendary" equipment. There is no single object of the game in World of Warcraft, although most players greatly enjoy advancing through the levels. At level 60 there is an emphasis on acquiring rare equipment which can take months of play.

Players establish guilds which are named groups that socialize and play together. Guilds can be designed to create somewhat customized play experiences. For example, an LDS (Church of the Latter Day Saints) guild stated on an online guild recruitment forum, "We welcome all players as long as they can respect our standards (i.e., no dirty language/swearing). If you wish to have fun in a clean gaming environment, contact us." There are religious-themed guilds, geography-based guilds, guilds for power gamers, guilds for casual players, large ambitious guilds, small intimate guilds. An important focus for many guilds is collecting reliable players for advanced play.

We have noted the geographic reach of World of Warcraft, indicating its cross-cultural appeal. We have not been able to find data on the gender, age, and ethnicity of players. However, we think it likely that World of Warcraft has attracted a more diverse group of players than most competitive video games for whom the typical player is a young male. Both authors joined guilds where they encountered many active female players as well as players over age 30. One author's guild was
"The Grand Threat," discovered in an online guild recruitment forum, and chosen for its gay-friendly status because of the author's interest in the GLBT (gay-lesbian-bisexualtransgender) community online. About a quarter of the active players in this guild were female. The other author was recruited in-game to "The Legion of Darkness." About one third of the active players in this guild were female. Player profiles from both guild websites and interview data revealed that female players included a 21-year old college student, a 23-year old divorced mother with a small child, a 39-year old mother who home schooled her children, a 43-year old social worker, a 37-year old writer from the Philippines, and a 50-year old attorney. Older male players included a father who played with his son, an IT manager, a bookstore manager, and an advanced graduate student. One of us interviewed and played with a player who did not belong to a guild, a man in his midfifties. We interviewed a married couple who played together. One level 60 player we interviewed reported that nine of the seventy players in his guild composed only of level 60 s were female. It is statistically improbable that we would have so frequently encountered female players and older players if they were extremely rare. We hope that Blizzard or another source will make available reliable census data or data from a large randomly constructed sample.

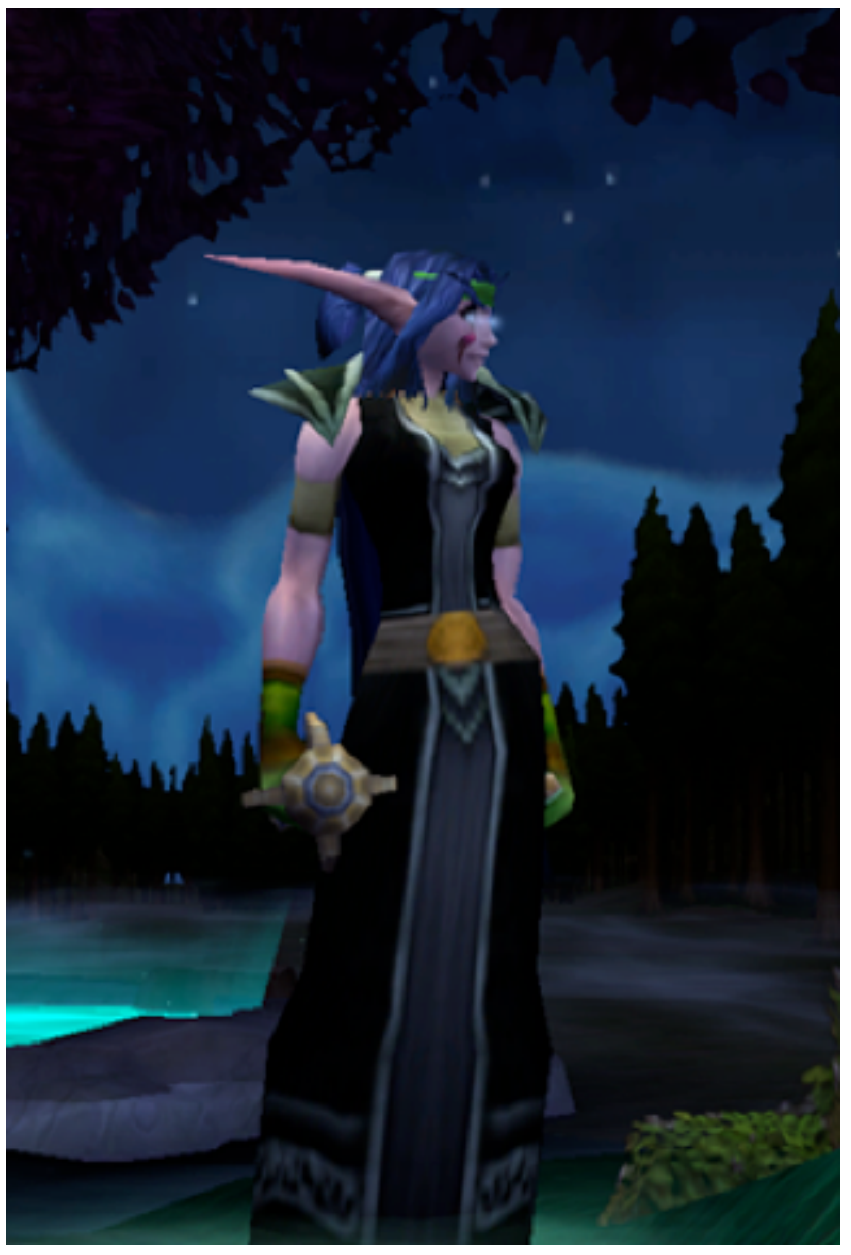

Figure One. A night elf priest. 


\section{METHODOLOGY}

We studied World of Warcraft through immersive ethnographic fieldwork including participant-observation; semi-structured in-depth interviews offline and online (through an in-game chat channel or email); the collection of chat logs (using a function supplied in the game); and reading documents such as WoW-related forums and websites. Late in 2005, we each created a character and logged on several times a week, playing intensively through the Christmas break, with ongoing play and observation continuing. One of us chose a druid and the other a priest for our characters. To gain as much knowledge of the game as possible, one of us joined a "player vs player" server and the other a "player vs environment" server. In player vs player, or PvP, players can attack and kill other players of the opposing "faction," Alliance or Horde. On PvE servers, players must intentionally flag themselves for PvP combat. PvP and PvE play are about evenly divided in number of characters [33]. Each of us experimented with alternative characters, or "alts," to gain further understanding of the classes and explore parts of the world inhabited by the two factions. We also observed two players creating and leveling new characters as well as advanced play in a level 60 guild, watching play for many hours. We conducted 26 indepth, semi-structured interviews, twelve with our guild members, eight with players we met and interviewed offline, and six with players we met online who were not in our guilds. Nineteen players were male and seven female. We asked people when and why they started to play WoW, how they learned the game, what they liked and disliked about it, how many characters they had, details regarding the characters, and whether they played with offline with friends and family. All guild and player names used are pseudonyms. Orthography in chat logs is unaltered.

\section{TYPES OF COLLABORATION IN WORLD OF WARCRAFT}

In this section we describe types of collaborative play in World of Warcraft ranging from fleeting lightweight encounters to highly structured collaborations in guilds. In section 4.1 we point out collaborations that take place with strangers to support Brown and Bell's [3] argument regarding the ease of collaborating with strangers in at least some MMOGs. In 4.2 we discuss the formal structures for collaborative support provided by game mechanics. In 4.3 we describe playful non-structured collaborations. In both, friends and strangers may collaborate.

\subsection{Strangers in the Fight}

A new player is suddenly surprised by a soft whooshing noise. A colorful icon appears near the top of her screen, and she experiences a moment of alarm. Was that a game-generated attack? No - the player has been "buffed," that is, she is the recipient of a beneficial spell cast by a nearby player. A buff increases an ability for a short period of time so that while in combat the player has a small advantage. Buffs are the simplest form of engagement between players we observed in World of Warcraft. While they are frequently used in formal groups, buffs are also a gesture of goodwill cast in passing on players with whom the caster has no relationship. Buffs are part of the culture of the game in which players commit small acts of kindness to maintain a mutually beneficial atmosphere even though no immediate reciprocity is in the offing and no rewards such as experience points are gained.

A "kill assist" is another common favor. While traveling, a player notices someone about to expire while battling a monster. Instead of continuing on, he stops to help make the kill, leaving a grateful player who avoids a dreary run back from the graveyard (the penalty for "death" in WoW). Or, a player has died before help could arrive. A nearby player who can return others to life resurrects the player.

Players offer assistance in other ways. A player finds himself in hostile territory with monsters too high-level for him to fight. He asks the help of a more advanced player and is escorted to safety-which can take several minutes and is a generous gesture. A frequent favor is to answer questions players ask in a chat channel. Players are constantly in need of information on how to play. For example, a player sends a message for the location of a particular computer character, receiving a reply giving directions.

It is likely that players in these encounters do not know one another. Players routinely offer unsolicited help to strangers as well as responding when asked. Because characters are labeled with their name and guild, and mousing over the character reveals class and level, players have enough information to make appropriate requests. A collaborative atmosphere of "respect" for all players is a desired aim articulated by many guilds and reinforced as players help others, mindful of help they have received.

Not all interactions with strangers are beneficial. A level 20 runs through a contested area on a PvP server and suddenly a mounted character appears on the road before him. The level 20 clicks on the character only to see a skull icon next to her name-WoW's way of saying that a fight with this person will surely bring death. As the first hit from the opponent drains half the character's life, he runs but is quickly defeated.

The level 20, now recovered, is in a close fight with a monster. As the moment of victory is reached he breathes a sigh of relief, only to be attacked from another quarter. A level 20 from the opposing faction has decided to take advantage of his weakened state for an easy kill. These activities are called "ganking." Not only are they allowed by game mechanics, they are considered fair play, as explained on the Blizzard website.

Unlike offline strangers, in the World of Warcraft unknown others are more than just part of the landscape. Players actively pursue engagements with them, both beneficial and combative. These informal, unplanned collaborations with other players bring added depth to the game as players fight their way through the computer-generated world.

\subsection{Structured Collaborations with Friends and Strangers}

World of Warcraft provides several means of structured collaboration where much play takes place. Players may play with players they don't know, with friends and family from offline life, or with in-game friends.

\subsubsection{Parties, raids, and the friends list}

Parties and raids are temporary group formed to accomplish a short-term goal such as a quest or defending against the opposite faction in PvP. They have a leader who invites players and can remove them. When conducting quests there 
are specific means by which experience points and treasure, or "loot," are shared so members know in advance what to expect.

A party, composed of 2-5 players, has its own chat channel. Party members may or may not know one another. For example, a party may set out at half-strength with players who know each other. They begin a quest and then add new members as needed as they meet other players doing the same quest. If party members enjoy playing together they may share their other quests. Quests are normally obtained from a computer character but quest-sharing allows players to continue to play together after the initial quest that brought them together is complete. If they like one another, players add each other to their friends list for future play.

The friends list is maintained individually by each player and contains a collection of people who usually do not know one another; they are known individually to the maker of the list. The friends list is one-way; adding a friend does not reciprocally add the player to the other's list. The friends list alerts the player when a friend logs in or out and provides information on their current level and location. Friends often "whisper" to one another, that is, communicate in a private chat channel. Friends are not weak ties as posited by Granovetter [13] because they typically do not link to other parts of a network as weak ties do, but remain one-to-one relations.

For bigger challenges than can be handled by parties, raids are formed. A raid is a group of up to eight parties. Each party within the raid has its own chat channel and a raid channel connects the members of the entire raid. Usually at least some raid members know one another. New members may also be added. WoW facilitates finding other players to party or raid with through a chat channel where people advertise the quests they wish to do. As with "pick-up groups" where parties are formed in the field, the chat channel can bring together players who do not know one another.

\subsubsection{Guilds}

Guilds are long-term groups which range in size from a small handful to a couple hundred players [9]. Players can belong to only one guild. There may be no specific goal for a guild other than for players to have a group to identify with. Or guilds may be highly organized and goal-driven (especially at higher levels). There is a guild chat channel and hierarchical ranks within the guild. Middle-level ranks can invite new members. This is a trusted responsibility because allowing the wrong people into a guild can ruin its social dynamics. The highest ranks can remove members, post messages that guild members see when they log in, and promote members to higher ranks. Guilds often organize guild-only raids and guildmates often group together. Much of the sociable non-game-related chat takes place in the guild channel. Most is informal, humorous, or downright silly although in one of the author's guilds, chat occasionally turned to more serious topics such as news reports of a player in China who had died because he did not get up from his keyboard. ("If we see anyone online that much, we'll stage an intervention," said the social worker in the guild.) Often players would report on their local weather, remark that they had a test to study for, or give other small details that revealed something of their lives. Over time, personal details about family, significant others, and so on, entered the conversation. Though such messages were brief, they provided enough information so that guildmates had a sense of others' lives. Friends engaged in similar chat. Many guilds have websites with player profiles, sometimes with photos, so guildmates have another source of information about one another.

\subsubsection{Battlegrounds}

Battlegrounds are places where structured PvP campaigns are waged. Players enter a queue and then join a team to play a game such as capture the flag. PvE players can also enter battlegrounds where they will be flagged $\mathrm{PvP}$ for the duration of the battle. While the goal of the game is to capture the flag (or similar goals), players can kill each other during play. Players can get experience points for quests conducted during play. Friends and guildmates may form a team or strangers are assigned teams.

\subsubsection{Duels and trades}

Duels and trades involve one-to-one engagements. In duels, players in the same faction challenge each other to fight but not to the death. One player formally issues a challenge and the other player can accept or decline. Duels are common even on PvE servers. Duels may take place between strangers or friends.

Players trade raw materials that have been collected with the gathering professions, as well as crafted items, enchantments, and loot they may not want. Trades can be accomplished through the trade chat channel or the Auction House where players put items up for bid. Players can also trade directly with one another through a trade window. Usually trading partners do not know one another and are brought together through the trade channel or Auction House.

In sum, World of Warcraft enables strangers and friends to collaborate through several formal mechanisms that establish a baseline of expectations about how play will be conducted; provide means of allowing players to communicate to accomplish their goals; provide structures for enabling transactions, such as the trade window; and organizing others, such as keeping track of friends, party members, and guildmates.

\subsection{Random Acts of Fun}

We return in this section to impromptu, less structured collaborations in World of Warcraft because they are interleaved with more structured collaborations, giving the game its sense of delight and unpredictability. When not engaged in battle, players come together to flirt, dance, drink, hug, joke, smile, laugh, and cheer. These actions, and many others, are supported by several dozen typed emote commands. For example, a player with a character named Annina types "/dance." The prompt says "Annina bursts into dance" and Annina begins a gender- and race-specific dance. Each race has a distinctive dance-slinky, earthy, humorous, ghoulish. Chances are Annina has joined a group of dancers carousing outside an inn or on the village green. Someone may have whispered, "Come dance with us." A group of players of different races and levels dancing together provides a welcome bit of levity after a difficult quest or narrow escape.

Players have fun in creative ways probably not envisioned by Blizzard designers. For example, we observed someone "teasing" a large dragon from the countryside into Stormwind, one of the major cities, and from there into the Auction House. 
Despite the upheaval of a large beast in a confined space where monsters are not intended to go, players were amused. We observed one level 60 who enjoyed inciting others to join him in riding in circles on their mounts in the busiest part of Ironforge, another major city, their enchanted weapons glowing and throwing off colorful sparkles. Guilds may host activities such as treasure hunts and parties. Figure Two shows characters on the way to a "slumber party," using the /follow command in an unusual way to form what players called a "conga line." Such amusements are part of player-created culture that makes use of game resources in unexpected ways. (See [9] for discussion of the "spectacles" WoW players create.)

These entertainments are intensified by the freer atmosphere common in online interactions [24] where the strictures of daily life are loosened. For example, one evening in Northshire Abbey we observed a group of characters who had discarded their clothing and were dancing in their undergarments in a fountain outside the Abbey. Such occurrences are rare offline but not in the fantasy play of WoW. Players may flirt or dance with same-sex players (regardless of the gender of the character). Both of us have observed such interactions in guild settings where we know the actual gender and sexual orientation of the player and we know they are known to the respective players because they have been revealed in guild chat or the players are offline friends. In such encounters gender doesn't matter the same way it does offline, and a less inhibited ambience prevails.

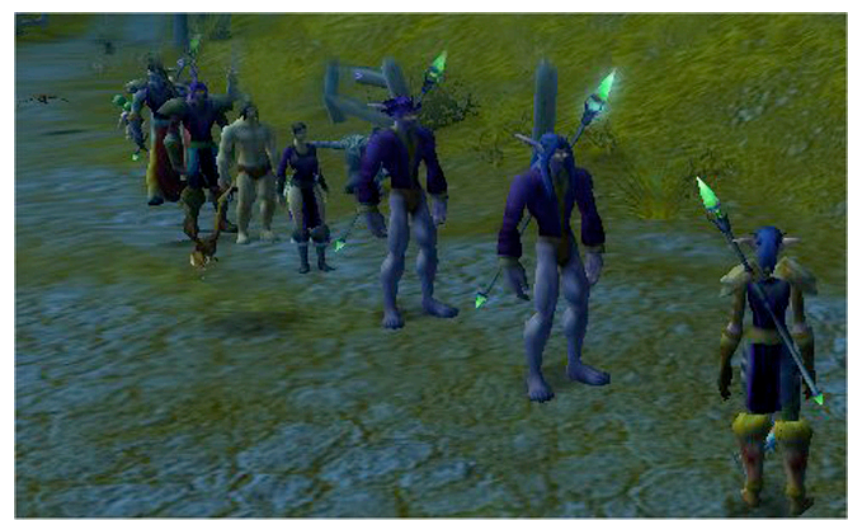

Figure Two. A conga line on the way to a guild party.

\subsection{Misbehaving in WoW}

Because our analysis focuses on collaboration, it may misleadingly suggest a constant friendliness that does not exist in the game. WoW generally maintains a congenial atmosphere, but there are a number of player actions that upset other players and are the source of player complaints. We have discussed ganking. While it is fair play according to Blizzard, ganking is annoying to players, as is "corpse camping." In PvP, a player may kill another player and "camp" the corpse, remaining by the corpse and killing the player after he resurrects and is in a weakened condition, sometimes repeating the action to the intense frustration of the defeated player. One player we talked to was paid to avenge a lower level player whose corpse had been camped by repeatedly killing and camping the corpse of the first attacker. Other causes for complaint are spamming chat channels with trade items or the use of mocking language in a general chat channel. One particular area, The Barrens, is known for such language in its channel. Calling someone's chatting behavior "Barrens chat" indicates that others view them as misbehaving.

\section{OFFLINE SOCIAL CONNECTION THROUGH WORLD OF WARCRAFT}

While arguments about social life on the Internet have suggested that it may lead to social isolation $[15,20,23]$, or, at best, weak social ties [7], it is common for WoW players to play with offline friends and family. Playing together includes grouping, sharing items, chatting, and exploring. Out of the 26 people we interviewed 20 currently played with offline friends and/or family and two others had in the past. Observations in both guilds showed this to be common among those not interviewed. Our interview sample may report a higher percentage of play with family and friends than actually exists because two informants were married to each other and two others played together in a guild.

WoW promotes offline social connection by providing a shared activity. College roommates and friends often play together (we had many more offers of interviews from college students who played together than we could handle in the first round of interviews). Friends who live in different cities may keep their friendships going in part by playing together. One player we interviewed had a set of eight friends in different parts of the Western U.S. with whom he played. Another played with his good friend in Germany, despite time zone differences. Both devoted players, they also had intense longdistance phone conversations about WoW. Nightflower, one of our guildmates who is a 53-year-old mother of seven, said in an online interview, "my oldest son just started playing last week and i am thrilled! he lives in NC and we haven't been close for a while...but now we talk a lot more ig and irl [ingame and in real life]." Another mother in the guild, when asked if playing with her children changed anything said, "Not really, we do a lot of things together." For her, playing WoW with her children was simply another shared activity. One player, who had two siblings and a nephew in the guild, said that they often used a voice chat program to talk while they played. It was, "like all being in the same room playing for a few hours a day." She also reported that she had a brother who played with his wife and their 9- and 14-year-old daughters on a role playing server, recreating the family through the characters! In the other guild, a mother of two who home schooled her children used WoW as part of the curriculum to study typing and math. The married couple we interviewed played together and had chosen character names, "Toast" and "Jam," to identify themselves as linked. We observed this playful naming convention in other family groups as well.

Another avenue of increased social connection is that WoW can be a topic of offline conversation for people who play together, and even for those who play, but not together. One level 60, an officer of The Grand Threat, related that he had gotten his brother to start playing, "and as a result we have new things to talk about, like in-game stuff." Another player, introduced to the game by his brother who was several years older, said, "Now we finally have something to talk about."

This is not to say that WoW necessarily always provides increasing offline social connection. Players sometimes value the ability to get away from offline relationships through the game. Halbarde said, "i think [playing WoW] has made me less 
social in real life. i will quite often pass on doing things to stay home and play. however, that isn't a bad thing, as before, i was quite the opposite and was having to learn to be by myself and enjoy my own company." Nightflower saw play as a way to reconnect with her children, but she also sometimes used the game to get a break. She said that offline she only had friends that she was very close to. Her in-game friends were refreshingly casual. Even then she said, "and to be honest sometimes i don't even want to do that [be tied to people in game] so i have a couple of horde char on another server that are not guilded that i play all alone."

\section{SOCIAL ORGANIZATION IN WORLD OF WARCRAFT}

We have described the many kinds of collaboration in World of Warcraft, grounding the discussion in the particularities of the game. We can analyze the collaborations in three categories: (1) communities, (2) "knots" [10], and (3) pairwise collaborations with friends. We will argue that two key game activities - having fun and learning the game-are enhanced by actions carried out in these arenas of collaboration, each with its own advantages. Having multiple arenas of collaboration, rather than just one, such as a community, provides a versatile, robust environment for play and learning.

\subsection{Communities}

We consider guilds to be communities. While the term "community" has been used in myriad ways [14], we adopt Driskell and Lyon's formulation that a community involves "common ties" and "social interactions" [7]. Common ties include a shared interest, bonds, commitment, a set of shared values, a culture, history, and shared identity [7]. Since World of Warcraft was only released in November 2004, guild histories may be rather short, but guilds, which clearly have the other elements of common ties, as well as rich social interactions, appear to us to be communities.

\subsection{Knots}

Engeström et al. [10] defined "knots" as unique groups that form to complete a task of relatively short duration. Examples of knots are an airline crew or personnel in a courtroom. Knots may also bring together strangers who spontaneously voluntarily agree to collaborate [10]. In knots strangers "tie a knot" to accomplish something together, as opposed to teams where members know one another and have persistent relationships. Parties in WoW are often knots (of the voluntary sort). So are battleground teams, trading partners, duelists, those who ask and answer questions in chat channels, and players who spontaneously cavort together.

Here is a partial transcript of a chat log of a typical instance of a knot in World of Warcraft. It shows portions of an hour's play between a priest and a hunter, both level 29, who form a party. They approach a dungeon at the same time, each alone. They have the quest "Insane Druids" which requires slaying Taneel Darkwood, Uthil Mooncall, and Mavoris Cloudsbreak. (The players switch from a local area chat to party chat at about 21:38.)

1/8 20:59:41.690 To Delbarth: are you doing insane druids?

1/8 21:02:13.481 Delbarth says: do you want to party up?

1/8 21:02:20.599 Delbarth has invited you to join a group.
1/8 21:02:50.556 Delbarth says: what point in the quest are you at?

1/8 21:03:02.604 Annina says: starting

1/8 21:03:12.441 Annina says: how about you?

1/8 21:03:24.256 Delbarth says: I have killed two of them need taneel still

1/8 21:03:30.935 Annina says: ok

1/8 21:03:32.444 Delbarth says: lets give it a go :)

1/8 21:03:35.112 Annina says: $\mathrm{k}$

[many monsters are slain including finally taneel]

1/8 21:38:11.186 [Party] Annina: ok got taneel!

1/8 21:38:11.809 [Party] Delbarth: oh yeah!

1/8 21:38:37.388 [Party] Delbarth: I am done with the quest, but you want to keep going?

1/8 21:38:44.612 [Party] Annina: yes!

[several monsters later]

1/8 21:41:41.345 [Party] Delbarth: you ok with mana?

1/8 21:41:46.243 [Party] Annina: yes

1/8 21:43:53.959 [Party] Delbarth: DING

1/8 21:44:00.636 [Party] Delbarth: 30th - wooh!

1/8 21:44:05.665 [Party] Annina: hurray!

[yet more monsters]

1/8 21:54:00.856 [Party] Delbarth: shall we keep going?

1/8 21:54:06.858 [Party] Annina: yes

[Annina gets one more of the monsters she needs]

1/8 21:55:32.578 [Party] Delbarth: thanks for the healing :)

1/8 21:56:46.311 [Party] Delbarth: I need to stop for the night - my wife is getting ancy :)

1/8 21:57:03.302 [Party] Annina: ok. thanks a lot for helping. and congrats on 30

1/8 21:57:20.881 [Party] Delbarth: thanks for the help, as well - can't do that dungeon solo, for sure!

The play continued well past the time Delbarth completed the quest and appeared to be interrupted by offline responsibilities. During the quest an exciting event occurred-Delbarth reached level 30, which he and Annina celebrated. At two points during play, Delbarth offered to continue even though he already had what he needed. He inquired about Annina's mana (the energy needed for casting spells) and they exchanged other pleasantries (not shown in the transcript) such as discussing his hunter's pet and the loot dropping from the monsters. By forming a knot, they collaborated to accomplish their goals and enjoyed a typical WoW experience. WoW makes such collaborations easy and the spirit of adventure and mutual aid created by players makes them fun.

\subsection{Pairwise collaborations with friends}

The friends list enables players to keep in touch with friends by being notified when they come online and go offline. Players may include a few guild members on their list if they want to be especially attentive to when they log in, although typically those on the friends list are not in the guild because there is a separate window showing guild member status. Players chat with friends, they seek and give advice, and may 
group with them. Friends occasionally arrange a meeting if they haven't seen each other's characters for awhile to check out new gear or to give each other gifts using the trade window. Gifts may also be sent through the mailbox system which only allows one-to-one mailings.

\section{DISCUSSION}

\subsection{A Multiplicity of Interleaved Collaborations}

Participation in a guild, collaboration in knots, and pairwise collaborations with friends lend the game variety, novelty, and surprise. These interleaved collaborations create a richly textured space in which play flows between community-based and lighter weight collaborations. One player with several characters including two level 60s said, "I like that you kind of feel a part of this bigger world. You have to go and explore and find out things and meet people."

At level 60, much play involves highly organized collaborations with well-known guildmates (see [28]). But even at this level, WoW players still engage in the lightweight collaborations of knots and friends that are part of the fabric of the game. For example, when asked about his favorite part of the game, Peter, a level 60 officer in his guild, said, "I have a lot of favorite things, especially selling enchantments. I like negotiating with other people and trying to make the most profit." He enjoyed advertising enchantments on a chat channel and haggling with other players to get the best price for his enchanting services.

Community-based relations in guilds are desirable and sought in the game. But the closer relationships can lead to what is often referred to as "drama." We asked Peter what he disliked about WoW. "Sometimes a lot of drama comes up," he replied. For example, a player may get upset if one of his friends is not invited into the guild, even though the guild cannot accommodate a player of his class. Or a player may be disruptive, running at a monster in what should be a carefully orchestrated attack, ruining play for everyone else. ${ }^{1}$ At the time of this writing, The Grand Threat was having problems with a lack of opportunities for level 60 play. Members were torn between seeking a new guild with others at their level and loyalty to the current guild. The Legion of Darkness experienced a wrenching change of leadership as the founding leader left to start a new guild, taking several good players with him. One day he simply disappeared, upsetting remaining players.

Such dislocations are part of guild life as we discovered in our own guilds and heard from informants in interviews. Driskell and Lyon [7] observed that it seems we never cease to pine for the tightknit communities putatively found in the small villages of Europe in the nineteenth century. Such villages were the inspiration for Tönnies's concept of Gemeinschaft-the intimate, close relations of a group of people who know one another well and share history and tradition [29]. But MMOGs provide an occasion to reflect on whether such communities should be the gold standard [7]. While Gemeinschaft-style social groups provide deep,

\footnotetext{
${ }^{1}$ This particular disruption was made famous in WoW lore in a humorous video called Leeroy Jenkins, available online. Peter reported events of this type happened in his guild.
}

supportive bonds, such bonds can be constraining as well as fulfilling. People may feel constrained to certain relationships and specific roles that may not actually suit them [1]. In WoW, the guild community is important for many, but not the only choice for collaboration. Many players value their friends list which represents an ego-centered personal social network in which a player's friends may know one another but usually do not. One player who was not in a guild said, "[I like] meeting good people online and being able to put them on my friends list." None of the players on his list knew one another. A player active in her guild said she still made other friends: "I have around fifteen on the list, three of whom are very good friends by now who I regularly talk to in-game." WoW makes it easy for players to establish different kinds of relationships, to have both a base in a guild and the opportunity to continue to interact with new players and to collaborate in varied ways.

As suggested by the Legion of Darkness's troubles, guild communities are often fragile. Driskell and Lyon noted that online communities are less robust than offline communities [7]. Many in our study reported that they had been in several guilds, which, for one reason or another, did not work out. The normalcy of collaborating with strangers means that finding others to collaborate with is not as difficult as it may be in more Gemeinschaft-like environments. Even Horde and Alliance may collaborate with tasks such as kill assists though they can communicate with one another only through emote commands.

Many players, including those in guilds, spend considerable time "soloing" in individual play, or playing in parties with non-guildmates (before level 60). They reported that they enjoy soloing because it is challenging and the overhead of collaborating with others is reduced (for example, it can easily take 30 minutes for players to assemble to enter a dungeon together). But soloers often find temporary partners, as discussed, and they chat with their friends, so the game is still a social experience. Another striking phenomenon is what one player called "alt-itis," that is, creating many alternative characters. Instead of heading for the highly organized play of "endgame," that is, play at level 60 , players begin a new character, or several. One player with two level 60 characters said, "I really feel like at level 60 the game has kind of lost its luster a little bit." He enjoyed the accomplishment of leveling and the less organized collaborations although he had also participated in lengthy raids with his level 60 characters and was familiar with that kind of play. Some players try a new form of play such as moving from PvE to PvP to head off endgame. Another player with level 60 characters enjoyed helping lower level players, giving advice or aiding a difficult quest.

WoW's interleaved collaborations advance the object of playing a game to have fun. That WoW is fun is expressed in players' wry use of the word "addicting" to describe the game, a word we heard repeatedly in interviews and one used so often on game forums that it is a cliché. As one of our guildmates said, "If only real life was this addicting." While players like many things about WoW such as leveling, virtually all of our informants mentioned the social aspects of WoW as crucial to their enjoyment. A level 46 mage said, "[WoW] is fun on many levels, and it's like a real world because you know you are playing with other real human beings rather than AI.... It's addicting." Another player said, "I like the social aspects of WoW and playing with other people gives it an added unpredictability." In the following interview segment, 
Dreadlock, a young male player leveling quickly toward endgame, connected the fun of WoW to opportunities for meeting people. (The :D icon means "grin." Instances are quests in "private" dungeons where only the party or raid members are present.)

To Dreadlock: what do you like about the game?

Dreadlock whispers: its fun :D

To Dreadlock: right! but why?

Dreadlock whispers: cant feal lonly while playing it!

Dreadlock whispers: to meet people, etcw

Dreadlock whispers: i love instances

To Dreadlock: why do you love them?

Dreadlock whispers: because they make it so you MUST group

Dreadlock whispers: its not an option

To Dreadlock: how do you find people to group with?

Dreadlock whispers: advertising in the chat channels, guild and asking my friends

Dreadlock whispers: also instances are an oppertuniy to meet people

Ducheneaut et al. described World of Warcraft as a game in which people spend significant amounts of time alone, in the presence of others, but not interacting with them [9]. Using logging software, they measured sociability as time spent grouping in parties, raids, and battlegrounds. Because these are only a portion of many social activities in WoW, it appeared that players were "alone" much of the time since grouping accounted for $30-40 \%$ of play.

However, the totality of collaborative play should be analyzed. Our analysis enumerated the many different kinds of collaborative play in WoW including parties, raids, friends, guilds, duels, battlegrounds, trades, as well as informal collaborations such as kill assists, answering questions, impromptu dancing and flirting, chatting, and PvP play. Taken together, these activities comprise a sociable experience, as our informants reported and we observed in-game. Raw time is a problematic metric for capturing sociability. Duels, for example, take only a short time but offer an enjoyable challenge and one that provides the stuff of stories players tell each other. Players may spend only a few minutes dancing on the village green, but those few vivid minutes are the difference between a single player game and a multiplayer game such as World of Warcraft.

Perhaps most important, chatting is a key aspect of socializing in WoW that is not captured in the metric used in [9]. Chatting takes place when grouping, but also while people are soloing, crafting, spending time in cities, or traveling. For example, traveling in WoW is time consuming, but many put their character on "auto-run" and chat while moving on foot around Azeroth, or while traveling on the slow transportation network of boats, trams, air monsters, and zeppelins. In fact, much sociable chat takes place during exactly such times when people are not in battle. Ducheneaut et al. argued that grouping was an obvious metric for sociability in World of Warcraft, but chatting and the multiplicity of collaborations we reported are central to game experience. The metrics in [9] also cannot capture the sociability of play between family members and offline friends. The authors suggested that offline friends who play together may get "separated" as they level at different rates, but it is common to have alts which the logging cannot account for. Because of alts, offline friends may still play together, providing a highly social experience. We had several such cases among our informants. Friends at different levels continue to chat. And a lower level player may request advice or assistance from a higher level friend. If offline friends and family spend $30-40 \%$ of game time grouping together, we might interpret this metric to suggest that such time together is considerable, intensifying already close social relationships.

We emphasize that much socializing in WoW is brief, lightweight, informal fun. At the same time, as play progresses, many players spend more and more time in dungeons which require groups. Our informants mentioned the satisfaction of "teamwork" in these groups. Ducheneaut et al. found that grouping increased as players progressed through the levels [9]. Players who do not enjoy socializing have many other games from which to choose. We do not believe it is an accident that a game that combines competition with many kinds of sociability is so enormously popular.

\subsection{Learning}

We have discussed the many ways WoW players collaborate to have fun. The interleaved collaborations in WoW also have important implications for learning. As with most MMOGs, there is a lot to learn. For example, players must understand different spells and when to use them; have some comprehension of the different classes so that when playing with others the character is positioned correctly and acts in a way that complements others' abilities; be able to predict how monsters will react; and know which equipment is best for the specific way the character has been created and for different contexts of battle (to mention only a few aspects of the game).

Guildmates are an important resource for learning. But nearly all players also ask strangers for help in chat channels or if a player is standing nearby. They may ask players on the friends' list, using their personal social networks. One player said, "I was trying to find my way into the Badlands and couldn't so I asked one of my friends and she explained how to. I don't know how long it would have taken me to find it on my own." Playing with others in temporary groups is often very instructive. For example, while grouping with a paladin at a quest in a cemetery, other players may learn that the paladin is especially effective against undead targets. The other players learn to adjust their actions to his special abilities and can use the knowledge they gain in other settings. In the interviews, players often referred to "trial and error" learning; playing with others is part of such learning.

Gamers' use of learning resources such as websites and forums has been well-documented $[11,27]$. The players in our study consulted sites such as Thottbot, Allakhazam, the Blizzard website and forums, online guides to playing a particular class, and various FAQs. A player can find finely detailed descriptions of game minutiae such as how to use a fishing pole or the way lower level players can make their way to the large cities when traveling through dangerous territory. Several sites provide useful user interface modifications or "mods," (e.g., CTMod) that many in our study took advantage of. Based on their own experiences, players write guides, FAQs, and mods well-matched to players' needs. 
In WoW, the individual learner coordinates the diverse resources in the collaboration space. The learner has agency in choosing and deploying learning resources-learning is not controlled by an outside agent such as an organization, peer, teacher, curriculum, or community. Learning occurs when the learner needs and wants it, in the context of solving a problem the learner genuinely wishes to solve (see [5]). A multiplicity of collaboration types offers redundant sites of learning. The opportunity to receive an answer to a question or obtain advice quickly and in context does not depend on the requirement that a player draw on Gemeinschaft relations as in, for example, apprenticeship learning (see [17]). Resources are distributed, and easily accessed by the learner who can seek advice and information from in-game friends and strangers, online sources, guildmates, and often offline friends and family. The atmosphere of mutual benefit that begins with the player's first buff provides an environment in which players have established an ethos of helping and asking for help. Players who write guides and contribute to forums found on the Internet extend this ethos outside the game, to online venues that are greatly valued by many players.

Vygotsky spoke of the "zone of proximal development" in which a learner advances by being offered a challenge and the appropriate resources to meet the challenge. The resources are supplied by a teacher or more experienced peers [31]. The zone is the difference between what the learner can do with and without the aid of the teacher or more experienced peers. In WoW, the zone of proximal development is unusually flexible because aid from more experienced peers is available from so many sources. While it is not possible here to undertake a detailed comparison to other learning environments such as traditional classrooms, apprenticeships, or online tutorials, we cannot think of another context of learning with the access and flexibility we observed within World of Warcraft and its associated online resources.

\section{DESIGN IMPLICATIONS}

Brown and Bell suggested that multiplayer games may have significant social benefits [3]. While World of Warcraft is pure entertainment, we believe its social organization comprised of communities, knots, and pairwise collaborations with friends has implications for other domains. We discussed the learning environment afforded by a mixed collaboration space of different types of collaborations. Such learning environments could be created for training and education in many fields. Contemporary learning theory has moved beyond the individual learner to understanding learning as collaboration in communities [17]. Our analysis of learning in WoW suggests that community is only one resource for learning. The multiplicity of resources beyond community gives learners the freedom to solve problems at just the right moment and to choose resources that suit them. Our research also suggests that it is not only the cognitive aspects of game design that provide learning advantages (as discussed in [11]) but WoW's specific social organization and the culture of mutual aid created by players. We do not believe games are a magic bullet for education by any means (see $[4,26]$ ) but we think that World of Warcraft provides an interesting model for learning a complex set of skills through its multiple sources of collaboration and spirit of "strangers in the fight."

Pearce noted that "boundaries between play and production, work and leisure...are increasingly blurring" [21]. Mixed collaboration spaces, whether MMOGs or another format, may be useful in domains such as interdisciplinary scientific work where a key challenge is finding the right collaborators [36]. Apart from locating a colleague in the appropriate discipline, issues of work style and personality play a role in successful collaborations. WoW gives people a chance to engage in lightweight collaborations and then assess whether they wish to continue the relationship in chat, as friends, or perhaps even in a guild. This notion of "trying on" brief collaborations in cyberspace might be fruitful in assembling remote teams comprised of people who have not had the opportunity to work together. Such collaboration spaces would contain meaningful tasks for the given domain that could be completed quickly, to give potential collaborators a sense of how they work together.

We can envision designing mixed collaboration spaces for eldercare, other groups with limited mobility, and corporate team building and training. Loneliness, lack of social support, and increased immobility have been shown to lead to depression in the elderly [22]. A game such as World of Warcraft tailored to the interests of various segments of the elderly population would enable them to make new friends online from their own homes, in the context of an exciting, challenging game with rich content. The game would provide mental stimulation and topics of mutual interest. Mental stimulation and social engagement reduce the risk of dementia [32]. Just as informants told us WoW was a subject of discussion with friends and family with whom they played, even those who played on different servers, games could provide elders with fresh material for conversation and a chance to meet new people. Other populations with limited mobility such as hospitalized children might similarly benefit. Corporate team building and training could be enhanced by suitably designed MMOGs, providing better benefits than a day at the bowling alley or online tutorials that do not rouse much enthusiasm.

Observers such as Putnam [23] have worried about loss of community in the modern context, negatively evaluating lightweight online relationships, referring to them as "driveby." But we have found that such relations in World of Warcraft make the game more fun and engender a flexible learning space. Driskell and Lyon suggested that while fullblown Gemeinschaft community is unusual in online collaboration, it might be reasonable to at least look for "Gemeinschaft-like relationships" [7]. We would look past even Gemeinschaft relationships to make visible the full spectrum of collaborations in a mixed collaboration space such as World of Warcraft. We propose that, taken together, the multiplicity of different kinds of collaborations creates a versatile, engaging space for collaboration. We found that rather than Gemeinschaft necessarily always being the ideal, multiple levels of collaborations, including those with strangers, also yield pleasure and new knowledge.

\section{CONCLUSION}

Social activity in World of Warcraft challenges discourse that asserts that the Internet leads to isolation or is simply community moved online. A space of mixed collaborations provides variety in play experience and flexibility in learning. Our findings suggest that lightweight collaborations can be enjoyable and enlightening and need not be negatively valued. The design of World of Warcraft and the player culture 
that have developed within the game provide an innovative space in which strangers collaborate and can become friends. At the same time, WoW joins a long tradition of card and board games in which family and friends of different ages and genders may play together.

\section{REFERENCES}

[1] Bargh, J., McKenna, K., and Fitzsimons, G. Can you see the real me? Activation and expression of the "true self" on the Internet. Journal of Social Issues 58 (2002), 33-48.

[2] Blizzard Entertainment Inc.. www.blizzard.com/press/050720.shtml www.blizzard.com/press/060119.shtml

[3] Brown, B. and Bell, M. CSCW at play: 'There' as a collaborative virtual environment. Proceedings $\mathrm{CSCW}$ 2004, ACM Press, New York, 350-359.

[4] Clegg, A. Games and simulations in social studies education. Handbook of Research on Social Studies Teaching and Learning, J. Shaver, ed. Macmillan, New York, 1991.

[5] Dewey, J. Experience and Education. Macmillan, New York, 1938.

[6] Dourish, P. The state of play. Journal of Computersupported Cooperative Work 7 (1998), 1-7.

[7] Driskell, R. and Lyon, L. Are Virtual Communities True Communities? Examining the Environments and Elements of Community. City and Community 1 (2002), 373-390.

[8] Ducheneaut, N. and Moore, R. The social side of gaming: A study of interaction patterns in a massively multiplayer online game. Proceedings CSCW 2004, ACM Press, New York, 360-369.

[9] Ducheneaut, N., Yee, N., Nickell, E., Moore, R. “Alone Together?" Exploring the social dynamics of massively multiplayer online games. Proceedings CHIO6, ACM Press, New York, 407-416.

[10] Engeström, Y., Engeström, R., and Vähäaho, T. When the center doesn't hold: The importance of knotworking. In S. Chaiklin, M. Hedegaard and U. Jensen (eds.). Activity Theory and Social Practice. AarhusPress, Denmark, 1999.

[11] Gee, J. What Video Games have to Teach Us about Learning and Literacy. Palgrave Macmillan, New York, 2003.

[12] Golder, S. and Donath, J. Hiding and revealing in online poker games. Proceedings CSCW 2004, ACM Press, New York, 370-373.

[13] Granovetter, M. The strength of weak ties. American Journal of Sociology 78 (1973), 1360-1380.

[14] Hillery, G. Definitions of community. Rural Sociology 20 (1955), 779-791.

[15] Kraut, R., Patterson, M., Lundmark, V., Kiesler, S., Mukhopadhyay, T. and Scherlis, W. Internet paradox: A social technology that reduces social involvement and psychological well-being? American Psychologist 53 (1998), 1001-31.

[16] Kushner, D. Engineering Everquest. IEEE Spectrum Online. July, 2005. http://www.spectrum.ieee.org/jul05/1561
[17] Lave, J. and Wenger, E. Situated Learning: Legitimate peripheral participation. Cambridge University Press, Cambridge, 1991.

[18] Leontiev, A. (1974). The problem of activity in psychology. Soviet Psychology 13 (1974), 4-33.

[19] Muramatsu, J. and Ackerman, M. Computing, social activity, and entertainment: A field study of a game MUD. Journal of CSCW 7 (1998), 87-122.

[20] Nie, N.H. and Erbring, L. Internet and Society: A Preliminary Report. IT \& Society 1 (2002), 275-283.

[21] Pearce, C. Productive play: Game culture from the bottom up. Games and Culture 1 (2006), 17-24.

[22] Prince, M., Harwood, R., Blizard, R., Thomas, A., and Mann, A. Social support deficits, loneliness and life events as risk factors for depression in old age. Psychological Medicine 27 (1997), 323-332.

[23] Putnam, R. Bowling Alone: The Collapse and Revival of American Community. Simon and Schuster, New York, 2000.

[24] Reid, E. Electropolis: Communication and Community on Internet Relay Chat. Honours Dissertation, University of Melbourne, 1991.

[25] Schiesel, S. The game is a hit but the work isn't done. New York Times. February 7, 2005.

[26] Squire, K. Changing the game: What happens when video games enter the classroom? Innovate: Journal of Online Education 1 (2005).

[27] Steinkuehler, C. Cognition and Learning in Massively Multiplayer Online Games: A Critical Approach. Ph.D. Thesis, University of Wisconsin, Madison, 2005.

[28] Taylor, T. Power gamers just want to have fun?: Instrumental play in a MMOG. Proceedings $1^{\text {st }}$ DiGra Conference. University of Utrecht, Netherlands, 2003.

[29] Tönnies, F. Community and Society: Gemeinschaft and Gesellschaft. Michigan State University Press, $1887 / 1957$.

[30] Twidale, M., Wang, X., and Hinn, D. CSC*: Computer supported collaborative work, learning, and play. In Koschmann, T., Suthers, D., and Chan, T. (eds.) Proceedings, Computer Supported Collaborative Learning 2005. Lawrence Erlbaum, New Jersey.

[31] Vygotsky, L. Mind and Society: The Development of Higher Mental Processes. Harvard University Press, 1978.

[32] Wang, H., Karp, A., Winblad, B., and Fratiglioni, L. Latelife engagement in social and leisure activities is associated with a decreased risk of dementia. American Journal of Epidemiology 155 (2002), 1081-1087.

[33] http://www.warcraftrealms.com/census.php

[34] http://www.worldofwarcraft.com/misc/awards.html

[35] Yoon, J., Oishi, J., Nawyn, J., Kobayashi, K. and Gupta, N. FishPong: Encouraging human-to-human interaction in informal social environments. Proceedings CSCW 2004, ACM Press, New York, 374-377.

[36] Zimmerman, A. and Nardi, B. Whither or Whether HCI: Requirements Analysis for Multi-Sited, Multi-User Cyberinfrastructures. Proceedings CHI 2006, ACM Press, New York 
